\title{
Simulations of a non-Markovian description of nucleation
}

\author{
J. Kuipers ${ }^{1, a)}$ and G. T. Barkema ${ }^{1,2}$ \\ ${ }^{1}$ Institute for Theoretical Physics, Utrecht University, 3584 CE Utrecht, The Netherlands \\ ${ }^{2}$ Instituut-Lorentz, for Theoretical Physics, Leiden University, 2333 CA Leiden, The Netherlands
}

(Received 24 June 2009; accepted 13 April 2010; published online 13 May 2010)

\begin{abstract}
In most nucleation theories, the state of a nucleating system is described by a distribution of droplet masses and this distribution evolves as a memoryless stochastic process. This is incorrect for a large class of nucleating systems. In a recent paper [J. Kuipers and G. T. Barkema, Phys. Rev. E 79, 062101 (2009)], we presented a non-Markovian model for droplet growth that includes memory effects and this model was treated analytically in the absence of a free energy landscape. In this paper, the model is considered with a free energy barrier present. Nucleation rates are measured in the prototypical example of nucleation in the Ising model. Results of direct simulations and the non-Markovian theory agree within a factor of 2 for spin-flip dynamics, and within $20 \%$ for local spin-exchange dynamics, even though the measured nucleation rates vary over 27 orders of magnitude. (C) 2010 American Institute of Physics. [doi:10.1063/1.3425732]
\end{abstract}

\section{INTRODUCTION}

Nucleation is an activated process that is key to understanding various subjects in polymer physics, biophysics, and chemistry. ${ }^{1}$ It has been studied extensively and the first theoretical description was given by Becker and Döring ${ }^{2}$ in the first half of the previous century. This classical nucleation theory (CNT) describes how stable nuclei spontaneously emerge in a metastable environment. The state of a nucleating system is described by a distribution $n(m, t)$ of droplet masses at time $t$. This distribution evolves via the Fokker-Planck equation $^{3}$ as a Markovian, i.e., memoryless, stochastic process.

In a recent paper, ${ }^{4}$ we presented an alternative model for droplet growth in nucleating systems, with the incorporation of memory effects as main characteristic. Although the underlying nucleating system with all degrees of freedom is typically evolving via Markovian dynamics, the reduction to a limited set of variables [in the case of CNT, the distribution $n(m, t)]$ gives rise to memory effects. This is most apparent in, e.g., nucleation in binary mixtures of fluids. Here, droplets of a certain type that have recently shrunk are surrounded by a higher density of particles of that type, and are therefore more likely to grow in the future. These memory effects are not part of CNT, but are included in our model. Memory effects like this are probably generic and also occur in other activated processes, such as protein folding, once the high-dimensional phase space is reduced to a lowerdimensional subspace or a reaction coordinate.

Our non-Markovian model for droplet growth has been analytically treated in the absence of a free energy barrier and it is shown that anomalous diffusion takes place for several orders of magnitude in time, i.e., the mean square cluster variation scales as $\left\langle\Delta m(t)^{2}\right\rangle \sim \sqrt{t}$. This behavior has been confirmed in simulations of the Ising model. The goal of this

\footnotetext{
${ }^{a)}$ Author to whom correspondence should be addressed. Electronic mail: jkuipers@phys.uu.nl.
}

paper is to perform numerical simulations of our droplet model in the presence of a free energy barrier and estimate the nucleation rate. This problem seems too hard to handle analytically. The free energy landscapes are provided by a three-dimensional Ising model with various dynamics and coupling constants. Together with simple a priori estimates for the dynamical parameters, the nucleation rates of our model are obtained. These are compared to the measured nucleation rates of the Ising model and the rates predicted by CNT with the Markovian assumption.

\section{CLASSICAL NUCLEATION RATE}

CNT describes the state of a nucleating system by a distribution $n(m, t)$ of droplet masses at time $t$. This distribution evolves via the Fokker-Planck equation

$$
\frac{\partial n(m, t)}{\partial t}=\frac{\partial}{\partial m}\left[R(m)\left(\beta \frac{\partial F}{\partial m}+\frac{\partial}{\partial m}\right) n(m, t)\right],
$$

with $R(m)$ as the rate at which droplets of mass $m$ grow to droplets of mass $m+1, F(m)$ as the free energy of a droplet of mass $m$, and $\beta$ as the inverse temperature. The free energy of a droplet is assumed to consist of two terms: a positive term proportional to its mass and a negative term proportional to its surface area. This free energy function gives rise to a critical droplet mass that maximizes the free energy. This explains why nucleation is an activated process. From Eq. (1), one can calculate the equilibrium distribution function

$$
n^{(\mathrm{eq})}(m)=\mathcal{N} e^{-\beta F(m)},
$$

with $\mathcal{N}$ a normalization constant, and the rate at which nucleation occurs by considering it as a steady current in the mass distribution with appropriate boundary conditions ${ }^{2}$

$$
\nu_{\text {nucl }}^{(\mathrm{CNT})}=\frac{1}{n^{(\mathrm{eq})}(1)}\left(\sum_{m=1}^{N} \frac{1}{R(m) n^{(\mathrm{eq})}(m)}\right)^{-1} .
$$




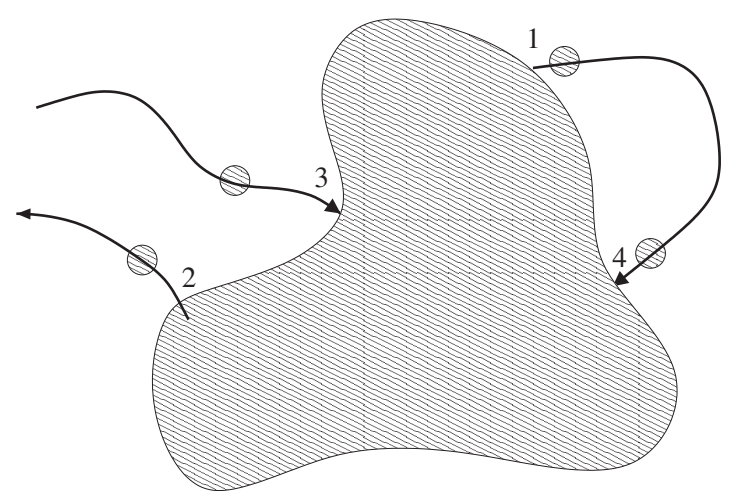

FIG. 1. Illustration of the four effects causing variations in the droplet mass in a dilute environment: (1) the emission of returning particles, (2) the emission the nonreturning particles, (3) the absorption of nonreturning particles, and (4) the absorption of returning particles.

\section{NON-MARKOVIAN DROPLET MODEL}

In our non-Markovian droplet model, ${ }^{4}$ we consider a droplet in a dilute environment. The droplet mass changes by means of four effects, namely, (1) the emission of particles from the droplet that are returning, (2) the emission of nonreturning particles, (3) the absorption of particles from far away (i.e., not returning from previous emission), and (4) the absorption of particles that are returning. These four effects are illustrated in Fig. 1. Effects 1-3 are independent and are described by three random functions $\xi_{r}(t), \tilde{\xi}_{n r}(t)$, and $\xi_{n r}^{+}(t)$, consisting of a series of Poisson distributed delta functions. The fourth effect, the absorption of returning particles, is correlated with the emittance of these particles. A particle emitted at time $\tau$ is returning with probability $p_{r}$. The return time is $T_{\tau}$, which is a random variable described by the return time distribution function $\mu$. This leads to the following stochastic differential equation describing the evolution of the mass $m(t)$ of a nucleating droplet:

$$
\dot{m}(t)=\xi_{n r}^{+}(t)-\xi_{n r}(t)-\xi_{r}(t)+\int_{-\infty}^{t} d \tau \delta\left(t-\tau-T_{\tau}\right) \xi_{r}(\tau)
$$

In Ref. 4, this equation is treated analytically for small deviations from the critical droplet size, i.e., in a flat free energy landscape. A typical nucleation process, however, takes place in the presence of a free energy barrier $F(m)$. This free energy is connected to a mass-dependent average systematic growth $v(m)$ of a droplet and a mass variation diffusion coefficient $D_{M}(m)$. The systematic growth and diffusion coefficient prescribe the expectation values of the random functions in Eq. (4) via the equations

$$
\begin{aligned}
& \left\langle\xi_{n r}^{+}(t)\right\rangle-\left\langle\xi_{n r}(t)\right\rangle=v(m(t)), \\
& \sum_{ \pm}\left\langle\delta \xi_{n r}^{ \pm}(t) \delta \xi_{n r}^{ \pm}\left(t^{\prime}\right)\right\rangle=2\left(1-p_{r}\right) D_{M}(m(t)) \delta\left(t-t^{\prime}\right), \\
& \left\langle\delta \xi_{r}(t) \delta \xi_{r}\left(t^{\prime}\right)\right\rangle=p_{r} D_{M}(m(t)) \delta\left(t-t^{\prime}\right),
\end{aligned}
$$

with $\delta \xi_{\alpha}(t) \equiv \xi_{\alpha}(t)-\left\langle\xi_{\alpha}(t)\right\rangle$. These equations result in the following expectation values of the random forces:

$$
\begin{aligned}
& \left\langle\xi_{n r}^{+}(t)\right\rangle=\left(1-p_{r}\right) D_{M}(m(t))-\frac{1}{2} v(m(t)), \\
& \left\langle\xi_{n r}(t)\right\rangle=\left(1-p_{r}\right) D_{M}(m(t))-\frac{1}{2} v(m(t)), \\
& \left\langle\xi_{r}(t)\right\rangle=p_{r} D_{M}(m(t)) .
\end{aligned}
$$

These three equations fix the properties of the three Poissonian random functions $\xi$ completely.

Our objective is to simulate Eq. (4) to estimate the nucleation rates of various three-dimensional Ising models with both spin-flip and local spin-exchange dynamics. For the systems, free energy landscapes are sampled. From the choice of dynamics, a priori estimates for the return probability $p_{r}$, the return time distribution $\mu$, and the mass fluctuation diffusion coefficient $D_{M}(m)$ are made. This defines all parameters of Eq. (4).

\section{ISING MODEL}

A prototypic system to study nucleation is the Ising model. ${ }^{5}$ To test our droplet model, nucleation rates are determined for a three-dimensional Ising model on a cubic lattice with periodic boundary conditions. The Hamiltonian is given by

$$
H=-J \sum_{\langle i, j\rangle} s_{i} s_{j}-h \sum_{i} s_{i},
$$

with the first sum over all pairs of adjacent sites.

The simulations are performed with two different types of dynamics: spin-flip and local spin-exchange. In the case of local spin-exchange dynamics, spin-flip moves are also performed in three strips of the system (one in each dimension). This mimics an infinite reservoir with a fixed density of minority spins and prevents depletion, otherwise caused by a nucleating cluster. Time is defined in units of spin-flip moves or spin-exchanges moves per site.

For both types of dynamics, the state spaces and Hamiltonians, and therefore also the free energy landscapes, are equal. This free energy landscape is needed in the definition of the non-Markovian droplet model. It is sampled with successive umbrella sampling, ${ }^{6}$ as function of the number $n_{6}$ of up-spins that have six aligned neighbors. This variable is chosen for multiple reasons. First, it indicates nucleation very well. Furthermore, it only changes with small increments per time step and is easy to update in the simulations. A typical free energy landscape is shown in Fig. 2.

\section{DROPLET MODEL PARAMETERS}

To complete the definition of our droplet model, assumptions are made for the mass fluctuation diffusion coefficient $D_{M}(m)$, the return probability $p_{r}$, and the return time distribution $\mu(t)$. Since the free energy $F$ has been sampled, the average systematic growth then follows from $v(m)$ $=D_{M} \beta \partial_{m} F(m)$.

For our droplet model describing spin-flip dynamics, $D_{M}(m)$ is chosen as 


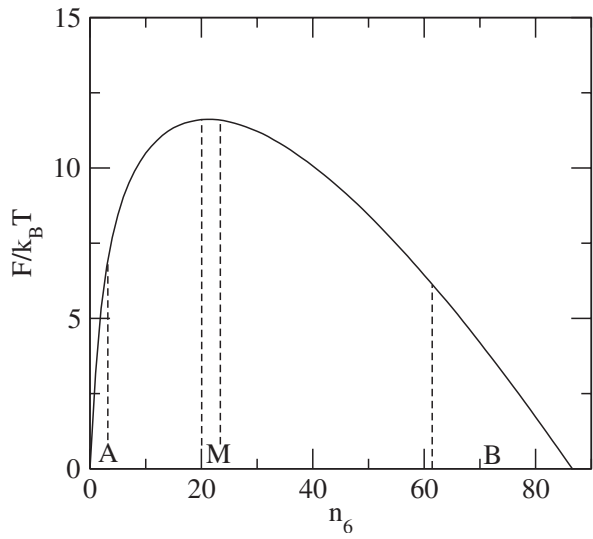

FIG. 2. Free energy landscape of the three-dimensional Ising model with coupling constant $\beta J=0.37$ and external magnetization $\beta h=0.2$. Also denoted are the prenucleation state $A$, the nucleated state $B$, and the region $M$ on top of the barrier, as used in the pathway recombination method.

$$
D_{M}^{(\mathrm{flip})}=f_{3} 4 \pi R_{\text {crit }}^{2}
$$

It is assumed that the critical cluster is spherical with radius $R_{\text {crit }}=\left(3 m_{c} / 4 \pi\right)^{1 / 3}$. Here, $f_{3}$ is the number of sites per unit surface area (around a sphere on a lattice), that touch three sites of the sphere. Spin flips at these sites do not increase the energy and therefore remain attached to the cluster. Spin flips at sites touching fewer sites of the cluster are energetically unfavorable and detach within times of order one. The return probability

$$
p_{r}^{(\text {flip })}=0
$$

since a flipped spin does not linger in the neighborhood of the cluster as is the case with a detached spin with local spin-exchange dynamics. The return time distribution $\mu^{\text {(flip) }}(t)$ is therefore irrelevant. This makes the model for spin-flip dynamics memoryless and basically identical to CNT. Instead of the ad hoc approximation for the mass fluctuation diffusion coefficient of Eq. (8), one could obtain a more accurate value by measuring it. Reference 7 presents a method to perform these measurements.

On the other hand, for local spin-exchange dynamics, this diffusion coefficient is chosen as

$$
D_{M}^{(\mathrm{exch})}=\pi R_{\mathrm{crit}}^{2} e^{-12 \beta J+2 \beta h} .
$$

Here, $\pi R_{\text {crit }}^{2}$ is the number of local spin-exchange moves per unit time that exchange a spin touching the critical cluster with a spin not touching it, and $e^{-12 \beta J+2 \beta h}$ is the density of single up-spins outside the cluster. This approximation only neglects the fact that the cluster is slightly nonspherical and the presence of small clusters in the surrounding environment, and is therefore expected to be very accurate. The return probability and return time distribution are determined by a three-dimensional random walk $^{8}$

$$
p_{r}^{(\mathrm{exch})}=\frac{R_{\mathrm{crit}}}{R_{\text {crit }}+\delta R}
$$

and

$$
\mu^{(\mathrm{exch})}(t)=\frac{\delta R \exp \left(-\delta R^{2} / 4 D_{B} t\right)}{\sqrt{4 \pi D_{B}} t^{3 / 2}} .
$$

Here $D_{B}=\frac{1}{2}$ and $\delta R=8+\pi^{2} / 8 \pi$, conform Ref. 4 .

\section{PATHWAY RECOMBINATION}

With all parameters defined, Eq. (4) can, in principle, be numerically integrated to obtain results. The free energy barriers, however, are typically very high, so that direct simulations do not yield useful results. Therefore, we resort to a variation in our pathway recombination $\operatorname{method}^{9}$ to efficiently sample nucleation trajectories. This method samples transition trajectories by starting on top of the barrier and going forward and backward in time until the attractors are reached. In the original method, these paths over the barrier are combined with paths around the attractors to generate complete transition trajectories and obtain transition times. In this paper, a variation on the pathway recombination method is used to sample nucleation rates instead of times.

Contrary to the computation of nucleation times, the nucleation rates could also be sampled with transition path sampling. ${ }^{10}$ Also, forward flux sampling ${ }^{11}$ could be used, if a notion of the history, i.e., the times at which returning particles were emitted, is taken into account. Other methods, such as milestoning, ${ }^{12}$ do not work, since they disregard the non-Markovianity of the dynamics.

To determine the nucleation rate, the free energy landscape of an Ising model is sampled and the two attractors $A$ and $B$ and a small region $M$ on top of the barrier are selected (see Fig. 2). Trajectories over the barrier are now generated by starting in a point in $M$ (randomly picked from the Boltzmann distribution) and going forward and backward in time until attractors are reached at both sides of the path. The free energy gap between the attractors and the top should be large, so that the system typically equilibrates in an attractor before reaching the top again.

While generating the trajectories, the time $T^{(M)}$ that one spends on average in $M$ is recorded and the numbers of paths $N_{X M Y}$ (with $X, Y=A, B$ ) are counted. From the free energy landscape it is known that the system should be in $M$ with probability $p^{(M)}$, and therefore

$$
n_{M}=\frac{p^{(M)}}{T^{(M)}},
$$

with $n_{M}$ as the rate at which trajectories through $M$ are traversed. The rate at which $A M B$-trajectories are traversed then equals

$$
n_{A M B}=\frac{N_{A M B}}{\sum_{X, Y} N_{X M Y}} n_{M} .
$$

The rate $\nu_{A B}$ from $A$ to $B$ obeys $p^{(A)} \nu_{A B}=n_{A M B}$, with $p^{(A)}$ the probability of being in $A$. Therefore, the transition rate equals

$$
\nu_{A B}=\frac{1}{p^{(A)}} \frac{N_{A M B}}{\sum_{X, Y} N_{X M Y}} \frac{p^{(M)}}{T^{(M)}} .
$$

This expression for the rate can be easily calculated from the quantities sampled during the simulations. 


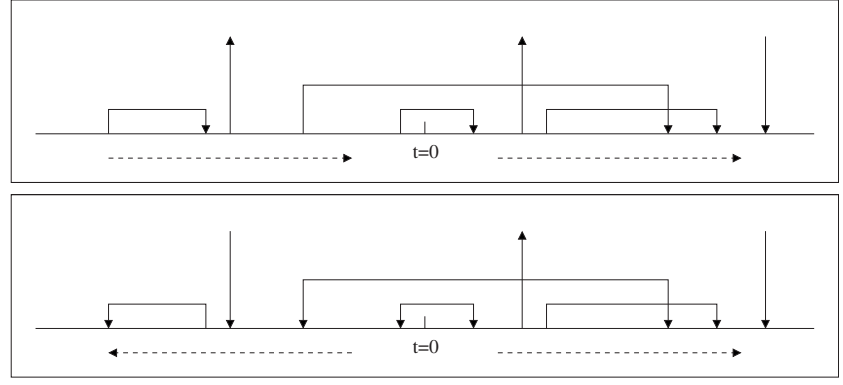

FIG. 3. Illustration of the pathway recombination simulations of our nonMarkovian droplet model. Top panel: a forward simulation. Bottom panel: an accompanying backward simulation. The solid arrows indicate absorption and emission of returning and nonreturning spins. The dashed arrows indicate the direction of time.

The simulations of the Ising model with pathway recombination are straightforward and performed analogous to the simulations in Ref. 9. The simulations of our droplet model, however, require careful treatment of the memory effects, especially since the simulations are going both forward and backward in time after starting in $M$, and those paths should be combined in a consistent way.

\section{PATHS IN THE DROPLET MODEL}

To sample paths in the non-Markovian droplet model, the forward simulations are performed first. Such a forward simulation is performed as follows: starting at time $t=0$ and going forward in time with increments of $\Delta t$, for every time interval the probabilities are calculated that a returning spin is emitted, or nonreturning spin is emitted or absorbed (effects 1-3 from Fig. 1). These probabilities are given by $\left\langle\xi_{r}(t)\right\rangle \Delta t,\left\langle\xi_{n r}(t)\right\rangle \Delta t$, and $\left\langle\xi_{n r}^{+}(t)\right\rangle \Delta t$, respectively. When a returning spin is emitted, a return time is randomly chosen from the return time distribution $\mu(t)$, and when the simulation arrives at that moment in time, a returning spin is absorbed. Returning spins emitted at times before $t=0$ are missing now. To include them, at every time the probability that a spin is returning, is calculated. This probability also equals $\left\langle\xi_{r}(t)\right\rangle \Delta t$. If a spin is returning, its emission time is randomly chosen from the distribution $\mu(t)$. If this time is larger than $t=0$, the returning spin is neglected, since spins returning from that time are already included in the simulation. If, on the other hand, this emission time is smaller than $t=0$, a returning spin is absorbed in the cluster. Figure 3 (top panel) depicts this simulation process. The forward simulation lasts until the cluster arrives in either the prenucleation state $A$ or the nucleated state $B$.

After the forward simulation has finished, a backward simulation starting with the same size at $t=0$ is performed. Since time is running backward, the change in cluster size $\dot{m}(t)$ gets a minus sign, so that absorption and emission are interchanged. In the forward simulations the emission times of spins emitted before $t=0$ are stored, and these times are used as return times in the backward simulations. In the backward simulations, this replaces the absorption of spins that are emitted between time $t=0$ and the end of the forward simulation. Except for this, the backward simulations are

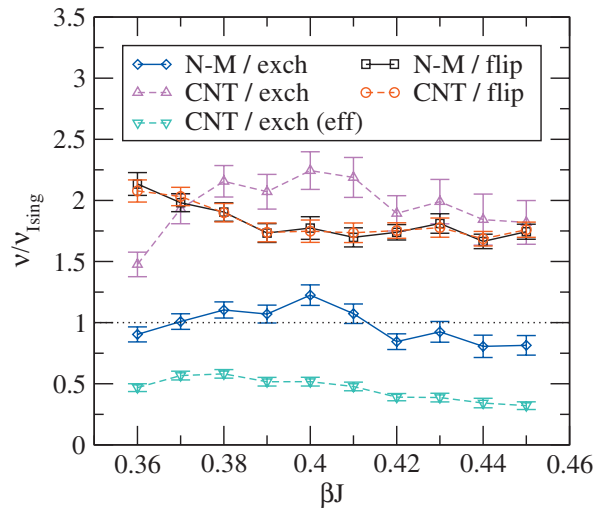

FIG. 4. Ratios of the predicted nucleation rates and the measured rates of the Ising model. The ratios are displayed for both the non-Markovian (N-M) droplet model and CNT, and both spin-flip and local spin-exchange dynamics. For CNT with spin-exchange dynamics, the results with diffusion coefficient $D_{M}^{(\mathrm{exch})}$ and effective diffusion coefficient $\left(1-p_{r}\right) D_{M}^{(\mathrm{exch})}$ are both displayed. A ratio of one indicates perfect agreement.

performed exactly like the forward simulations. Figure 3 (bottom panel) depicts this backward simulation process.

\section{RESULTS}

The simulations of the Ising model are performed with both spin-flip and local spin-exchange dynamics on a 32 $\times 32 \times 32$ cubic lattice with periodic boundary conditions. The fixed external magnetization is $h=0.2 k_{B} T$ and the coupling constant is in the range $J=(0.36-0.45) k_{B} T$. This leads to critical clusters of sizes varying from ten to 150 spins. For these cluster sizes, the return probability of a detaching particle is around $80 \%$. The obtained nucleation rates range from $10^{-5}$ to $10^{-32}$, thereby spanning dozens of orders of magnitude. For each parameter choice for the Ising model, our non-Markovian droplet model is also simulated with the appropriate parameters to estimate to nucleation rate.

The results of the simulations are shown in Fig. 4. For both spin-flip and local spin-exchange dynamics, the nucleation rates as predicted by our droplet model divided by the measured nucleation rates of the Ising model are shown. For spin-exchange dynamics, this factor is approximately 1 , showing that our method predicts the nucleation rate accurately. For spin-flip dynamics, this ratio is approximately a factor of 2. We consider this a fair agreement. The precise cause of this factor is unknown, but we consider the chosen diffusion coefficient of Eq. (8) as a likely source. Furthermore, memory effects due to the shape of a cluster might play a role.

Also shown are the rates as predicted by CNT with the Markovian assumption, i.e., Eq. (3), divided by the same measured rates of the Ising model. The calculation of these rates uses the same free energy landscapes and diffusion coefficients as the simulations of our droplet model. In the case of local spin-exchange dynamics, the results with diffusion coefficient $\left(1-p_{r}\right) D_{M}^{(\text {exch) }}$ are also shown, since one may argue that this is the effective large-time diffusion coefficient.

For spin-flip dynamics, the results of our model and CNT with the Markovian assumption coincide. This is as expected, since the absence of memory effects renders our 
model identical to the classical one. For local spin-exchange dynamics, the rates predicted by our model are much closer to the measured nucleation rates of the Ising model than the classical rates are. The results of CNT with the Markovian assumption with $D_{M}^{(\text {exch) }}$ as in Eq. (10) are too large and the results with the corrected effective $\left(1-p_{r}\right) D_{M}^{(\mathrm{exch})}$ are too small. Our non-Markovian model with memory effects provides estimations of nucleation rates which never deviates from directly measured rates by more than $20 \%$, even though these nucleation rates vary over many orders of magnitude.

\section{ACKNOWLEDGMENTS}

We thank Henk van Beijeren for useful discussion.

${ }^{1}$ D. Kashchiev, Nucleation: Basic Theory with Applications (Butterworth-
Heinemann, Oxford, 2000).

${ }^{2}$ R. Becker and W. Döring, Ann. Phys. 24, 719 (1935).

${ }^{3}$ N. G. van Kampen, Stochastic Processes in Physics and Chemistry (Elsevier, Amsterdam, 1981).

${ }^{4}$ J. Kuipers and G. T. Barkema, Phys. Rev. E 79, 062101 (2009).

${ }^{5}$ M. E. J. Newman and G. T. Barkema, Monte Carlo Methods in Statistical Physics (Oxford University Press, Oxford, 1999).

${ }^{6}$ P. Virnau and M. Mueller, J. Chem. Phys. 120, 10925 (2004).

${ }^{7}$ K. Brendel, G. T. Barkema, and H. van Beijeren, Phys. Rev. E 71, 031601 (2005).

${ }^{8}$ J. G. Wendel, Ann. Probab. 8, 164 (1980).

${ }^{9}$ J. Kuipers and G. T. Barkema, J. Chem. Phys. 128, 174108 (2008).

${ }^{10}$ C. Dellago, P. G. Bolhuis, and P. L. Geissler, Adv. Chem. Phys. 123, 1 (2002).

${ }^{11}$ R. J. Allen, P. B. Warren, and P. R. ten Wolde, Phys. Rev. Lett. 94, $018104(2005)$.

${ }^{12}$ A. K. Faradjian and R. Elber, J. Chem. Phys. 120, 10880 (2004). 